\title{
A Mathematical Model For Lassa Fever Transmission Dynamics With Impacts of Control Measures: Analysis And Simulation
}

\author{
Oke I. Idisi and Tunde T. Yusuf
}

\begin{abstract}
Lassa Fever, caused by Lassa virus, is a vectorhost transmitted infectious disease whose prevalence has been on the upsurge over the past few decades. Thus, considering the grave implications of the continuous spread of the disease, an epidemic model was developed to describe the disease transmission dynamics with impacts of proposed control measures. This is to help inform effective control strategies that would successfully curtail and contain the disease in its endemic areas. The model is qualitatively analyzed in order to contextualize the long run behavior of the model while the model associated basic reproduction number is derived. The model analysis reveals that the disease-free equilibrium is locally and globally stable whenever the basic reproduction number is less than unity and the disease prevalence would be high as long as the basic reproduction number is greater than unity. Finally, the model is numerically solved and simulated for different scenarios of the disease outbreaks while the findings from simulations are discussed.
\end{abstract}

Index Terms-Lassa virus, Vector-Host model, Basic reproduction number, Equilibrium solutions, Stability analysis.

\section{INTRODUCTION}

Lassa fever is an acute viral illness caused by the lassa virus. The vector for the virus are the rodents of the genus Mastomys, also known as the "multimammate rats". Lassa virus is of family Arenaviridae which includes Junin virus discovered in 1958, Machupo virus (1963), Gunarito virus (1989), Sabia virus (1993), Chapare virus (2004) and Lujo virus (2008). All these viruses have their members generally associated with rodent-transmitted diseases in humans. These viruses are zoonotic, hence do not obviously cause illness to their host [3], [13]. Lassa virus was first discovered in a town called Lassa located within Borno state in Nigeria during the year 1969 and it has since spread to other countries. For instance, It has been reported in Benin Republic, Côte d'Ivoire, Ghana, Liberia, Mali, Nigeria, Sierra Leone and Togo [3], [13]-[15]. In 2019, the Nigeria Center for Disease Control (NCDC) declared the outbreak of Lassa fever as emergency with 324 confirmed cases of Lassa fever and 72 death with case fatality rate (CFR) of $22 \%$ of the 3746 contacts identified in January 2019 across 20 states in Nigeria including the federal capital territory [20]. Recently, the Nigeria Center for Disease control (NCDC) cumulative

DOI: http://dx.doi.org/10.24018/ejmath.2021.2.2.17

Published on March 31, 2021.

Oke I. Idisi is with the Lagos State University, Nigeria.

Tunde T. Yusuf is with the Federal University of Technology, Nigeria. (e-mail: ttyusuf@futa.edu.ng). report from week 1 to week 41, 2020, recorded 228 deaths with a (CFR) of $20.7 \%$. Thus, the disease is gradually becoming endemic in Nigeria having spread across 27 states comprising 129 Local Government Areas of the federation. Moreover, it is imperative to mention that $80 \%-90 \%$ of humans contact Lassa fever either through primary human infection or secondary human infection route. The primary human infection occurs through eating food contaminated by infected rat (Mastomys Natalensis) urine/droppings while its secondary human infection occurs through direct contact with blood secretion, organs or other bodily fluids of infected persons. As for the rodents, the virus are passed from mother rodent to offspring during pregnancy and most infection are transmitted among adults rodent during sex, fighting, and bites. Also, the viruses are shed into the environment in urine or droppings of infected host [3], [13]. It is essential to mention that the incubation period of Lassa disease ranges between $6-21$ days and the prodromal of disease is associated with mild symptoms similar to that of Malaria and Typhoid fever. These symptoms include nausea, vomiting, chest pain, puffy face, puffy cheeks, edema, dehydration, conjunctiva injection, fainting attacks, bleeding from orifices, hypertension, coma, and shock development in severe cases. Nevertheless, Lassa fever can be treated using Ribavirin which is effective at the early stage of infection [12], [13], [15].

Over the years, mathematical models have been used to study vector-host disease transmission dynamics [11], [16]. For example; Esteva and Vargas, Cruz-Pacheco et al, Boureima et al (see [17]-[19]) developed and investigated compartmental models for the spread of dengue fever, West Nile virus and Malaria respectively; using a system of ordinary differential equations. Similarly, Brauer et al, and Cai \& $\mathrm{Li}$ developed a basic transmission mathematical model to study some vector-transmitted diseases (see [1], [10]). The Brauer et al study was centered on transmission dynamics and control of Zika virus disease while Cai and $\mathrm{Li}$ analyzed a simple vector-host epidemic model with direct transmission using SIS and SIRS compartments for the human population and SI for the vector population.

$\mathbf{I}$ $\mathrm{N}$ recent times, the emergence of Lassa fever has precipitated several researches on how to understand the dynamics of disease transmission and combat the spread of the disease. Consequently, Sulaimon and Ibrahim [5] 
formulated a mathematical model describing the transmission dynamics of Lassa fever infection. They adopted the SEIR compartment model for the human population with a split of the infectious class into symptomatic and asymptomatic infectious classes while assuming that rodents do not recover once they are infected with the Lassa virus. They defined a threshold parameter and concluded that when the respective basic reproduction number $\mathcal{R}_{h}<1$, the disease dies out and if otherwise, the disease persists in the population. Aside, there are quite a few other works on Lassa fever disease transmission dynamics (see [2], [23], [24]). Thus, authors like Omale \& Edibo, Akanni \& Adediipo, and Eraikhuemen \& Eguasa all developed and analyzed mathematical models for Lassa fever transmission dynamics with control strategies. They equally carried out stability analysis and simulations of their models while they proffer suggestions on control strategies that can help mitigate the alarming spread of the disease based on findings from their various studies (see [4], [6], [8]). The purpose of this paper is to describe and analyze a mathematical model for the transmission dynamic of Lassa virus within the vector and host population. Thus, the paper is structured as follows: In section 2, the proposed mathematical model for Lassa Fever transmission dynamics will be formulated; In Section 3, qualitative analysis of the model will be presented; In section 4, the model will be solved numerically and simulated for different scenarios of the disease outbreak while the simulation results will be discussed; In section 5, the conclusion from the study will be given.

\section{Proposed Model Formulation}

$\mathbf{I}$ $\mathrm{N}$ this section, a vector-host epidemic model with direct and indirect vector transmissions is presented and analyzed, the human population is divided into susceptible, exposed, infected and recovery (SEIR) compartments and the rodent population is divided into susceptible and infected (SI) compartments. Susceptible humans are recruited in to the population at rate $\Lambda_{H}$ and became exposed having contact with either infected humans or rodents at rate $\beta_{1}$ or $\beta_{2}$. Exposed individuals proceed to the infected compartment at rate $\gamma$ and die due to Lassa disease at rate $\kappa_{H}$, infected individual who recovered from the disease proceed to the recovery compartment at rate $a$, while $\mu$ denote natural death in each of the human compartment. Also, rodents are recruited into susceptible population at $\Lambda_{R}$ and became infected having sufficient contact at rate $\tau$ and they die either naturally or by human hunting denoted by $\phi_{R}$ or $\delta_{R}$. It is assumed that all humans are susceptible to the infection $a b$ initio.

The proposed model satisfies the following assumptions

1) Susceptible individuals can be infected via two routes of transmission, that is, directly, through a contact with an infected individual (supposedly as a result of blood transfusion,or sexual transmission), and indirectly through eating of contaminated food by the urine or feces of infected rodents. Thus, we denote the rate of direct transmission by $\beta_{2}$ so that the incidence of new infections via this route is given by a standard incidence rate $\frac{\beta_{2} I_{H} S_{H}}{N_{H}}$. also we denote the rate of indirect transmission of Lassa with $\beta_{1}$, and the incidence of new infections transmitted by the vectors is given again by a standard incidence rate $\frac{\beta_{1} I_{R} S_{H}}{N_{R}}$.

2) The total human population $N_{H}$ is heterogeneously mixed and the total rodent population $N_{H}$ is equally heterogeneously mixed.

3) It is assumed that, at the outset, there is no control measure in place to check the free spread of the disease.

Thus, the dynamics of this infectious disease in the human and rodent populations can be described using system of nonlinear differential equations Eq. (1) and the schematic diagram presented in Fig. 1 below:

$$
\begin{aligned}
\frac{d S_{H}}{d t} & =\Lambda_{H}-\frac{\beta_{1} S_{H} I_{R}}{N_{R}}-\frac{\beta_{2} S_{H} I_{H}}{N_{H}}-\mu S_{H}, \\
\frac{d E_{H}}{d t} & =\frac{\beta_{1} S_{H} I_{R}}{N_{R}}+\frac{\beta_{2} S_{H} I_{H}}{N_{H}}-\gamma E_{H}-\mu E_{H}, \\
\frac{d I_{H}}{d t} & =\gamma E_{H}-\kappa I_{H}-\alpha I_{H}-\mu I_{H}, \\
\frac{d R_{H}}{d t} & =\alpha I_{H}-\mu R_{H}, \\
\frac{d S_{R}}{d t} & =\Lambda_{R}-\frac{\tau S_{R} I_{R}}{N_{R}}-\phi S_{R}-\delta S_{R}, \\
\frac{d I_{R}}{d t} & =\frac{\tau S_{R} I_{R}}{N_{R}}-\phi I_{R}-\delta I_{R} .
\end{aligned}
$$

At any time, the total population of humans and rodents is given respectively, by

$$
\begin{aligned}
N_{H}(t) & =S_{H}(t)+E_{H}(t)+I_{H}(t)+R_{H}(t), \\
N_{R}(t) & =S_{R}(t)+I_{R}(t),
\end{aligned}
$$

subject to the following non negative initial conditions

$$
S_{0 H}>0, E_{0 H} \geq 0, I_{0 H} \geq 0, R_{0 H} \geq 0, S_{0 R}>0, I_{0 R} \geq 0
$$

The entire growth for human and rodent population is respectively described by the following equation:

$$
\begin{aligned}
& \frac{d N_{H}}{d t}(t)=\Lambda_{H}-\mu N_{H}(t)-\kappa I_{H}(t), \\
& \frac{d N_{H}}{d t}(t)=\Lambda_{R}-(\phi+\delta) N_{R}(t) .
\end{aligned}
$$

\section{Model Qualitative Analysis}

Here, the qualitative analysis of the model Eq. (1) is carried out to determine the eventual behavior of the system in the long run. In order to achieve this, firstly we show that the model is mathematically well pose and that its compact set is positively invariant. Secondly, the model equilibrium solutions are obtained and the criteria for their stability are established using Routh-Hurwitz criteria and direct Lyapunov function technique. 


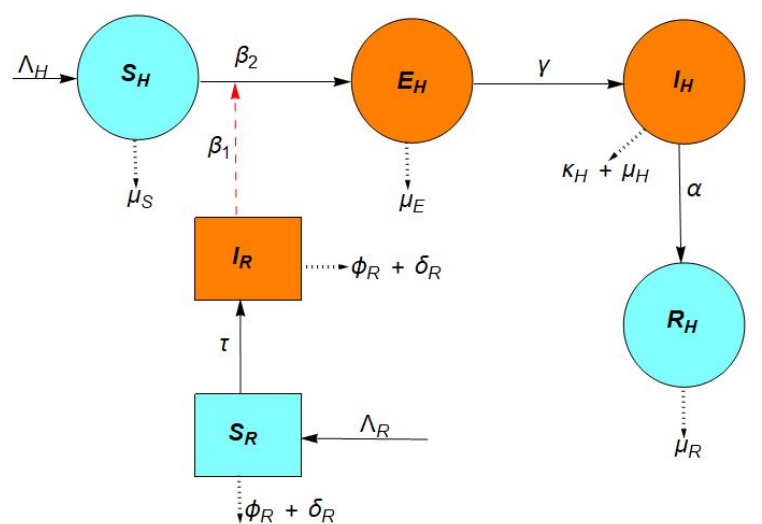

Fig. 1. Flow diagram for the spread of Lassa virus involving human and rodent transmission. Note that: Orange nodes are infectious and Aqua nodes are non-infectious. Circular shapes represents human cohorts and square boxes represent rodent cohort

TABLE I

DESCRIPTION OF VARIABLE AND PARAMETER FOR MODEL SYSTEM (1)

\begin{tabular}{l|l}
\hline Variable & Interpretation \\
\hline$S_{H}$ & Population of susceptible individuals \\
$E_{H}$ & Population of individuals exposed to Lassa \\
$I_{H}$ & Population of infectious individuals \\
$R_{H}$ & Population of individuals recovered from Lassa \\
$S_{R}$ & Population of Susceptible rodents \\
$I_{R}$ & Population of infectious rodent \\
\hline Paramater & Interpretation \\
\hline$\Lambda_{H}, \Lambda_{R}$ & Recruitment rates \\
$\beta_{1}, \beta_{2}$ & Transmission rates \\
$\gamma$ & Progression rate from $E_{H}$ to $I_{H}$ \\
$\alpha$ & Progression rate from $I_{H}$ to $R_{H}$ \\
$\tau$ & Progression rate from $S_{R}$ to $I_{R}$ \\
$\kappa$ & Disease induced death rate \\
$\phi$ & Death due to predator / human hunting of rodent \\
$1 / \delta$ & Rodent Average life span \\
$1 / \mu$ & Human average life span \\
\hline
\end{tabular}

\section{A. Positivity of Solutions for Model (1)}

Lemma 3.1: The compact $\Omega$ defined as $\left(\Delta_{1} \times \Delta_{2}\right)$ is a positively invariant set, which attracts all positive orbits in $\mathcal{R}_{+}^{6}$.

Based on system of Eq. (1), we have:

$$
\frac{d N_{H}}{d t}=\Lambda_{H}-\mu N_{H}-\kappa I_{H}
$$

Since $\kappa>0$ and $I(t) \geq 0$ for all $t \geq 0$, Eq. (5) becomes:

$$
\frac{d N_{H}}{d t} \leq \Lambda_{H}-\mu N_{H}
$$

Integrating Eq. (6), we obtained:

$$
\begin{aligned}
N_{H}(t) & \leq \frac{\Lambda_{H}}{\mu}+\left(N_{H}(0)-\frac{\Lambda_{H}}{\mu}\right) \mathbf{e}^{-\mu t} \\
& \leq N_{H}(0) \mathbf{e}^{-\mu t}+\frac{\Lambda_{H}}{\mu}\left(1-\mathbf{e}^{-\mu t}\right)
\end{aligned}
$$

Similarly, solving the second equation of Eq. (2):

$$
\begin{aligned}
N_{R}(t) & \leq \frac{\Lambda_{R}}{\phi+\delta}+\left(N_{R}(0)-\frac{\Lambda_{R}}{\phi+\delta}\right) \mathbf{e}^{-\mu t} \\
& \leq N_{R}(0) \mathbf{e}^{-\mu t}+\frac{\Lambda_{R}}{\phi+\delta}\left(1-\mathbf{e}^{-\mu t}\right)
\end{aligned}
$$

Therefore, as $t \geq 0, N_{H}(t) \leq \frac{\Lambda_{H}}{\mu}$ and $N_{R}(t) \leq \frac{\Lambda_{R}}{\phi+\delta}$ Hence:

$$
\begin{aligned}
\Delta_{1} & =\left\{\left(S_{H}, E_{H}, I_{H}, R_{H}\right) \in \mathcal{R}_{+}^{4}: N_{H} \leq \frac{\Lambda_{H}}{\mu}\right\} \\
\Delta_{2} & =\left\{\left(S_{R}, I_{R}\right) \in \mathcal{R}_{+}^{2}: N_{R} \leq \frac{\Lambda_{R}}{\phi+\delta}\right\} \\
\Omega & =\left\{\Delta_{1} \times \Delta_{2} \mid N_{H} \leq \frac{\Lambda_{H}}{\mu}, N_{R} \leq \frac{\Lambda_{R}}{\phi+\delta}\right\}
\end{aligned}
$$

This implies that $\frac{\Lambda_{H}}{\mu}$ and $\frac{\left.\Lambda_{R}\right)}{(\phi+\delta)}$ are the upper bond for human population $N_{H}(t)$ and rodent reservoir $N_{R}(t)$ respectively. Thus, from lemma 3.1 the set $\Omega$ is positive invariant with respect to system of Eq. (1). Then we, conclude that the set $\Omega$ is positively invariant and all solutions of Eq. (1) are non negative and epidemiological well posed.

\section{B. Reproduction number}

In order to further understand the disease transmission dynamics, we analyze the basic reproduction number $\mathcal{R}_{0}$, which is defined as the number of secondary disease cases caused by introducing a single infective individual during his/her infectious period into a wholly susceptible population of host and vector. For the proposed model Eq. (1), this is calculated using the approach in [1] which considers only human infections as new infections with infections of rodent as transition. It is salient to point out that the same expression was obtain for the reproduction number using the next generation matrix approach by [1] \& [21]. Hence, using the approach in ( [1] section 3), we form the matrix product as $K_{L}=F V^{-1}$ with

$$
F_{i}=\left[\begin{array}{c}
\frac{\beta_{1} S_{H} I_{R}}{S_{R}+I_{R}}+\frac{\beta_{2} S_{H} I_{H}}{S_{H}+E_{H}+I_{H}+R_{H}} \\
0 \\
\frac{\tau S_{R} I_{R}}{S_{R}+I_{R}}
\end{array}\right], V_{i}=\left[\begin{array}{c}
(\gamma+\mu) E_{H} \\
-\gamma E_{H}+(\kappa+\alpha+\mu) I_{H} \\
(\phi+\delta) I_{R}
\end{array}\right]
$$

such that $i=1,2,3$, thus the associated matrices $F$ denote new infectious terms and $V$ the remaining transition terms computed using the following Jacobian matrices:

$$
F=\left[\begin{array}{lll}
\frac{\partial F_{1}}{\partial E_{H}} & \frac{\partial F_{1}}{\partial I_{H}} & \frac{\partial F_{1}}{\partial I_{R}} \\
\frac{\partial F_{2}}{\partial E_{H}} & \frac{\partial F_{2}}{\partial I_{H}} & \frac{\partial F_{2}}{\partial I_{R}} \\
\frac{\partial F_{3}}{\partial E_{H}} & \frac{\partial F_{3}}{\partial I_{H}} & \frac{\partial F_{3}}{\partial I_{R}}
\end{array}\right], V=\left[\begin{array}{lll}
\frac{\partial V_{1}}{\partial E_{H}} & \frac{\partial V_{1}}{\partial I_{H}} & \frac{\partial V_{1}}{\partial I_{R}} \\
\frac{\partial V_{2}}{\partial E_{H}} & \frac{\partial V_{2}}{\partial I_{H}} & \frac{\partial V_{2}}{\partial I_{R}} \\
\frac{\partial V_{3}}{\partial E_{H}} & \frac{\partial V_{3}}{\partial I_{H}} & \frac{\partial V_{3}}{\partial I_{R}}
\end{array}\right]
$$

After proper substitution and evaluations of the matrices in (11) at the disease free equilibrium, we obtain:

$$
F=\left[\begin{array}{ccc}
0 & \beta_{2} & \frac{\beta_{1} \lambda_{H}(\phi+\delta)}{\mu \lambda_{R}} \\
0 & 0 & 0 \\
0 & 0 & \tau
\end{array}\right], \quad V=\left[\begin{array}{ccc}
\gamma+\mu & 0 & 0 \\
-\gamma & \kappa+\alpha+\mu & 0 \\
0 & 0 & \phi+\delta
\end{array}\right]
$$


Thus, the inverse matrix of $V$ in equation (11) was obtain as:

$$
V^{-1}=\left[\begin{array}{ccc}
\frac{1}{\gamma+\mu} & 0 & 0 \\
\frac{\gamma}{(\gamma+\mu)(\kappa+\alpha+\mu)} & \frac{1}{\kappa+\alpha+\mu} & 0 \\
0 & 0 & \frac{1}{\phi+\delta}
\end{array}\right]
$$

Since the first column of $F$ in equation(11) has zero entries, the same is true of $F V^{-1}$, and thus, the basic reproduction number $\left(\mathcal{R}_{0}\right)$ is the largest eigenvalue of $F V^{-1}$ which implies that $\mathcal{R}_{0}=\rho\left(F V^{-1}\right)=\max \left\{\mathcal{R}_{h}, \mathcal{R}_{r}\right\}$ where:

$$
\mathcal{R}_{h}=\frac{\beta_{2} \gamma}{(\gamma+\mu)(\kappa+\alpha+\mu)}, \quad \mathcal{R}_{r}=\frac{\tau}{(\phi+\delta)}
$$

where $\mathcal{R}_{r}$ and $\mathcal{R}_{h}$ is the reproduction number due to an infected rodents and infected human population respectively.

\section{Local Stability Analysis of $\mathcal{E}_{0}$}

The basic reproduction number serves as the threshold parameter in determining the relative rapidity of the spread of an infectious disease. Its value is also a measure of the ease or difficulty of controlling the spread of a disease. Thus, the bigger the value of $\mathcal{R}_{0}$, the more difficult it will be to curtail the spread of the disease in question, and vice-versa.

1) Disease Free Equilibrium: The disease free equilibrium $\left(\mathcal{E}_{0}\right)$ is the scenario where no individual and rodent are infected by Lassa virus in the country of interest. Mathematically this is determined by setting the derivatives $S_{H}^{\prime}=E_{H}^{\prime}=I_{H}^{\prime}=$ $R_{H}^{\prime}=S_{R}^{\prime}=I_{R}^{\prime}=0$ in system (1) and solving the resulting system of equations simultaneously. Thus, the model disease free equilibrium $\left(\mathcal{E}_{0}\right)$ is given as:

$$
\mathcal{E}_{0}=\left[\left(S_{H}, E_{H}, I_{H}, R_{H}, S_{R}, I_{R}\right)\left(\frac{\Lambda_{H}}{\mu}, 0,0,0, \frac{\Lambda_{R}}{\phi+\delta}, 0\right)\right]
$$

In addition, the stability of the disease-free equilibrium is established to reveal the conditions under which Lassa fever cases will die out or persist in the population.

Theorem 3.2: The disease free equilibrium $\mathcal{E}_{0}$ of system (1) is locally asymptotically stable if $\mathcal{R}_{0}<1$ and unstable if $\mathcal{R}_{0}>1$.

Proof: Evaluating the Jacobian matrix of Eq. (1) at the diseasefree equilibrium gives $J_{\left(\mathcal{E}_{0}\right)}=$

$$
\left[\begin{array}{cccccc}
-\mu & 0 & -\beta_{2} & 0 & 0 & -\frac{\beta_{1} \Lambda_{H}(\phi+\delta)}{\mu \Lambda_{R}} \\
0 & -\gamma-\mu & \beta_{2} & 0 & 0 & \frac{\beta_{1} \Lambda_{H}(\phi+\delta)}{\mu \Lambda_{R}} \\
0 & \gamma & -\alpha-\kappa-\mu & 0 & 0 & 0 \\
0 & 0 & \alpha & -\mu & 0 & 0 \\
0 & 0 & 0 & 0 & -\phi-\delta & -\tau \\
0 & 0 & 0 & 0 & 0 & \tau+-\phi-\delta
\end{array}\right]
$$

The characteristic equation $\left|J_{0}-I \lambda\right|=0$ is obtained from the Jacobian determinant with the Eigenvalues $\lambda_{i}(i=1, \ldots, 6)$ gotten as follows:

$$
(\lambda+\mu)^{2}(\lambda+\phi+\delta)(\lambda-\tau+\phi+\delta)\left[\begin{array}{cc}
-\gamma-\mu-\lambda & \beta_{2} \\
\gamma & -(\alpha+\kappa+\mu)-\lambda
\end{array}\right]=0
$$

From equation (17), either

$$
(\lambda+\mu)^{2}(\lambda+\phi+\delta)(\lambda-\tau+\phi+\delta)=0
$$

or

$$
\left[\begin{array}{cc}
-\gamma-\mu-\lambda & \beta_{2} \\
\gamma & -(\alpha+\kappa+\mu)-\lambda
\end{array}\right]=0
$$

Solving equation (18) to obtain the eigenvalues, we have:

$$
\begin{aligned}
& \lambda_{1}=-\mu, \quad \lambda_{2}=-\mu, \quad \lambda_{3}=-\phi-\delta, \\
& \lambda_{4}=\tau-\phi-\delta \quad \text { and } \quad \lambda_{4}=\left(\mathcal{R}_{r}-1\right)(\phi+\delta)
\end{aligned}
$$

Based on Routh-Hurwitz criteria, the matrix in equation (19) has a $\operatorname{trace}(A)<0$ and a $\operatorname{Det}(A)>0$ provided $\mathcal{R}_{0}<1$, since

$$
\text { Trace }(\mathrm{A})=-(\gamma+\mu+\lambda)-(\alpha+\kappa+\mu+\lambda)
$$

is obviously negative because all the parameters $(\gamma, \mu, \kappa$, and $\alpha)$ are positive and

$$
\begin{aligned}
\text { Det A } & =(\gamma+\mu)(\alpha+\kappa+\mu)-\beta_{2} \gamma, \\
& =\left(1-\mathcal{R}_{0}\right)(\gamma+\mu)(\alpha+\kappa+\mu) ; \\
& >0 \text { whenever } \mathcal{R}_{0}<1
\end{aligned}
$$

From Eq. (16) the first four eigenvalues all have negative real parts. We then establish the necessary and sufficient conditions for the remaining two eigenvalues of equation (19) to have negative real part. Therefore, if $\mathcal{R}_{0}<1$, then $\lambda_{5} \cdot \lambda_{6}>0$ and $\lambda_{5}+\lambda_{6}<0$. consequently, we have that the disease-free equilibrium $\left(\mathcal{E}_{0}\right)$ of the model is locally asymptotically stable if and only if the basic reproduction number $\left(\mathcal{R}_{0}\right)$ is less than unity. This complete the proof $\square$.

\section{Endemic Equilibrium $\left(\mathcal{E}_{1}\right)$}

System (1) can be shown to have a unique endemic equilibrium of the form $\left(S_{R}^{*}, I_{R}^{*}, S_{H}^{*}, E_{H}^{*}, I_{H}^{*}, R_{h}^{*}\right)$. Due to the complex nature of the model, system (1) was solved using mathematical software and the following results were obtained:

$$
S_{R}^{*}=\frac{\Lambda_{R}}{\tau}, \quad I_{R}^{*}=\frac{\Lambda_{R}(\tau-\delta-\phi)}{\tau(\delta+\phi)}=\frac{\Lambda_{R}\left(\mathcal{R}_{r}-1\right)}{\tau}
$$

$S_{H}^{*}=\frac{\Lambda_{H}\left[-\mu^{3} \tau-\chi \tau \mu^{2}-\tau \gamma\left(\alpha-\kappa+\beta_{2}\right) \mu-\xi \beta_{1} \tau+\beta_{1} \xi(\delta+\phi)+\sqrt{\Delta}\right]}{2 \gamma \mu\left(\tau\left(\beta_{2}-\kappa\right) \mu+\kappa(\delta+\phi-\tau) \beta_{1}\right)}$

$E_{H}^{*}=\frac{\Lambda_{H}\left[(\delta+\phi-\tau)\left(\mu^{2}+\chi \mu+\gamma(\alpha+2 \kappa)\right) \beta_{1}-\mu \tau\left(\mu \chi-\gamma\left(\beta_{2}-\chi\right)\right) \mu \tau+\sqrt{\Delta}\right]}{2 \gamma \mu\left(\kappa(\delta+\phi-\tau) \beta_{1}+\mu \tau\left(\beta_{2}-\kappa\right)\right)}$

$I_{H}^{*}=\frac{\Lambda_{H}\left[(\delta+\phi-\tau)\left(\mu^{2}+\chi \mu+\gamma(\alpha+2 \kappa)\right) \beta_{1}-\mu \tau\left(\mu \chi-\gamma\left(\beta_{2}-\chi\right)\right) \mu \tau+\sqrt{\Delta}\right]}{2\left(\tau\left(\beta_{2}-\kappa\right) \mu+\kappa(\delta+\phi-\tau) \beta_{1}\right) \chi(\gamma+\mu)}$

$R_{H}^{*}=\frac{\alpha \Lambda_{H}\left[(\delta+\phi-\tau)\left(\mu^{2}+\chi \mu+\gamma(\alpha+2 \kappa)\right) \beta_{1}-\mu \tau\left(\mu \chi-\gamma\left(\beta_{2}-\chi\right)\right) \mu \tau+\sqrt{\Delta}\right]}{2 \mu\left(\tau\left(\beta_{2}-\kappa\right) \mu+\kappa(\delta+\phi-\tau) \beta_{1}\right) \chi(\gamma+\mu)}$

with $\xi=\mu^{2}+(\alpha+\gamma+\kappa) \mu+\alpha \gamma, \quad \chi=\alpha+\kappa+\mu$,

$$
\left.\Delta=\mu^{2} \gamma+\mu\right)^{2} \chi^{2} \tau^{2}-2 \tau\left[\beta_{2} \gamma \mu \tau+(\delta+\phi-\tau) \xi \beta_{1}\right] .
$$$$
(\gamma+\mu) \mu \chi+\left[\beta_{2} \gamma \mu \tau-(\delta+\phi-\tau) \xi \beta_{1}\right]^{2}
$$ 


\section{E. Global Stability Analysis of DFE $\left(\mathcal{E}_{0}\right)$}

Theorem 3.3: The disease free equilibrium of Eq. (1) is globally asymptotically stable, if $\mathcal{R}_{0}<1$ and unstable if $\mathcal{R}_{0}>$ 1.

Proof:

Considering the quadratic Lyapunov function for the model Eq. (1):

$$
\begin{aligned}
W(t)= & \left(S_{H}-S_{H}^{*} \operatorname{In} S_{H}\right)+E_{H}+I_{H}+R_{H}+ \\
& \left(S_{R}-S_{R}^{*} \operatorname{In} S_{R}\right)+I_{R}
\end{aligned}
$$

Differentiating (22) with respect to time yields

$$
\begin{aligned}
\dot{W}(t) & =\dot{S}_{H}\left(1-\frac{S_{H}^{*}}{S_{H}}\right)+\dot{E}_{H}+\dot{I}_{H}+\dot{R}_{H}+\dot{S}_{R}\left(1-\frac{S_{R}^{*}}{S_{R}}\right)+\dot{I}_{R} \\
\dot{W}(t) & =\left(1-\frac{S_{H}^{*}}{S_{H}}\right)\left(\Lambda_{H}-\frac{\beta_{1} S_{H} I_{R}}{N_{R}}-\frac{\beta_{2} S_{H} I_{H}}{N_{H}}-\mu S_{H}\right) \\
& +\frac{\beta_{1} S_{H} I_{R}}{N_{R}}+\frac{\beta_{2} S_{H} I_{H}}{N_{H}}-(\gamma+\mu) E+\gamma E-(\kappa+\alpha+\mu) I_{H} \\
& +\alpha I_{H}+\mu R_{H}+\left(1-\frac{S_{R}^{*}}{S_{R}}\right)\left(\Lambda_{R}-\frac{\tau S_{R} I_{R}}{N_{R}}-(\phi+\mu) S_{R}\right) \\
& +\frac{\tau S_{R} I_{R}}{N_{R}}-(\phi+\mu) I_{R}
\end{aligned}
$$

Simplifying Eq. (23), we obtain:

$$
\begin{aligned}
\dot{W}(t) & =\Lambda_{H}\left(1-\frac{S_{H}^{*}}{S_{H}}\right)+\mu S_{H}^{*}\left(1-\frac{S_{H}}{S_{H}^{*}}\right)+\frac{\beta_{1} S_{H}^{*}}{N_{R}}\left(1-\frac{S_{H}}{S_{H}^{*}}\right) I_{R} \\
& +\frac{\beta_{2} S_{H}^{*}}{N_{R}}\left(1-\frac{S_{H}}{S_{H}^{*}}\right) I_{H}+\left[\frac{\beta_{2} S_{H}}{N_{H}}-(\kappa+\mu)\right] I_{H}-\mu E \\
& -\mu R+\left[\frac{\beta_{1} S_{H}}{N_{R}}+\frac{\tau S_{R}}{N_{R}}-(\phi+\delta)\right] I_{R}+\Lambda_{R}\left(1-\frac{S_{R}^{*}}{S_{R}}\right) \\
& +\frac{\tau S_{R}^{*}}{N_{R}}\left(1-\frac{S_{R}}{S_{R}^{*}}\right) I_{R}+(\phi+\delta) S_{R}^{*}\left(1-\frac{S_{R}}{S_{R}^{*}}\right)
\end{aligned}
$$

Using condition of Eq. (15), equation (24) can be expressed as:

$$
\begin{aligned}
\dot{W}(t) & =\Lambda_{H}\left(1-\frac{S_{H}^{*}}{S_{H}}\right)+\mu S_{H}^{*}\left(1-\frac{S_{H}}{S_{H}^{*}}\right)+\Lambda_{R}\left(1-\frac{S_{R}^{*}}{S_{R}}\right) \\
& +(\phi+\delta) S_{R}^{*}\left(1-\frac{S_{R}}{S_{R}^{*}}\right)-\mu E_{H}-\mu R_{H} \\
& \text { But } \quad S_{H}^{*}=\frac{\Lambda_{H}}{\mu} \quad \text { and } \quad S_{R}^{*}=\frac{\Lambda_{R}}{\phi+\delta} \\
\dot{W}(t) & \leq \Lambda_{H}\left(1-\frac{S_{H}^{*}}{S_{H}}\right)+\Lambda_{H}\left(1-\frac{S_{H}}{S_{H}^{*}}\right)+\Lambda_{R}\left(1-\frac{S_{R}^{*}}{S_{R}}\right) \\
& +\Lambda_{R}\left(1-\frac{S_{R}}{S_{R}^{*}}\right)-\mu E_{H}-\mu R_{H} \\
& \leq \Lambda_{H}\left(2-\frac{S_{H}}{S_{H}^{*}}-\frac{S_{R}^{*}}{S_{R}}\right)+\Lambda_{H}\left(2-\frac{S_{R}}{S_{R}^{*}}-\frac{S_{R}^{*}}{S_{R}}\right) \\
& -\mu E_{H}-\mu R_{H}
\end{aligned}
$$

$$
\dot{W}(t) \leq-\frac{\Lambda_{H}\left(S_{H}-S_{H}^{*}\right)^{2}}{S_{H} S_{H}^{*}}-\frac{\Lambda_{H}\left(S_{R}-S_{R}^{*}\right)^{2}}{S_{R} S_{R}^{*}}-\mu E_{H}-\mu R_{H}
$$

$\dot{W}(t) \leq 0$, only when $E_{H}, R_{H}=0$. Therefore, the maximum invariant set in is the singleton set $\mathcal{E}_{0}$. Hence, the global stability of $\mathcal{E}_{0}$ when $\mathcal{R}_{0}>1$ follows from LaSalle's invariance principle (see [25]). This means that the disease free equilibrium $\Theta=\left(S_{H}, E_{H}, I_{H}, R_{H}, S_{R}, I_{R}\right)$ is globally asymptotically stable on $\Omega \square$.

\section{F. Global Stability Analysis of $\mathcal{E}_{1}$}

Theorem 3.4: If $\mathcal{R}_{0}>1$, then there exist an endemic equilibrium $\mathcal{E}_{1}$ (in addition to the disease free equilibrium) of system (1) and it is globally asymptotically stable.

Proof. Suppose $\mathcal{R}_{0}>1$, then the existence of the endemic equilibrium point $\left(\mathcal{E}_{1}\right)$ is assured. Applying the common quadratic Lyapunov function

$$
V\left(x_{1}, x_{2} \ldots, x_{n}\right)=\left[\sum_{i=1}^{n} \frac{c_{i}}{2}\left(x_{i}-x_{i}^{*}\right)\right]^{2}
$$

Then, the derivative of $V$ with respect to time is given by:

$$
\begin{aligned}
\frac{d V}{d t}= & {\left[\left(S_{H}-S_{H}^{*}\right)+\left(E_{H}-E_{H}^{*}\right)+\left(I_{H}-I_{H}^{*}\right)+\left(R_{H}-R_{H}^{*}\right)+\left(S_{R}-S_{R}^{*}\right)\right.} \\
& \left.+\left(I_{R}-I_{R}^{*}\right)\right] \cdot\left[\frac{d\left(S_{H}+E_{H}+I_{H}+R_{H}+S_{H}+I_{H}\right)}{d t}\right]
\end{aligned}
$$

Substituting equation (5) into (26) yields:

$$
\begin{aligned}
\frac{d V}{d t}= & {\left[\left(S_{H}-S_{H}^{*}\right)+\left(E_{H}-E_{H}^{*}\right)+\left(I_{H}-I_{H}^{*}\right)+\left(R_{H}-R_{H}^{*}\right)+\left(S_{R}-S_{R}^{*}\right)\right.} \\
& \left.+\left(I_{R}-I_{R}^{*}\right)\right] \cdot\left[\Lambda_{H}+\Lambda_{R}-\mu\left(S_{H}+E_{H}+I_{H}+R_{H}\right)\right. \\
& \left.-\kappa I_{H}-(\delta+\phi)\left(S_{R}+I_{R}\right)\right]
\end{aligned}
$$

By Setting

$$
\begin{aligned}
\Lambda_{H} & =\mu\left(S_{H}^{*}+E_{H}^{*}+I_{H}^{*}+R_{H}^{*}\right)+\kappa I_{H}^{*} \quad \text { and } \\
\Lambda_{R} & =(\delta+\phi)\left(S_{R}^{*}+I_{R}^{*}\right)
\end{aligned}
$$

and substituting into equation (27), we have:

$$
\begin{aligned}
& \frac{d V}{d t} \leq {\left[\left(S_{H}-S_{H}^{*}\right)+\left(E_{H}-E_{H}^{*}\right)+\left(I_{H}-I_{H}^{*}\right)+\left(R_{H}-R_{H}^{*}\right)+\right.} \\
&\left.\left(S_{R}-S_{R}^{*}\right)+\left(I_{R}-I_{R}^{*}\right)\right] \cdot\left[\mu\left(S_{H}^{*}+E_{H}^{*}+I_{H}^{*}+R_{H}^{*}\right)+\right. \\
& \kappa I_{H}^{*}+(\delta+\phi)\left(S_{R}^{*}+I_{R}^{*}\right)-\mu\left(S_{H}+E_{H}+I_{H}+R_{H}\right)- \\
&\left.\kappa I_{H}-(\delta+\phi)\left(S_{R}+I_{R}\right)\right] \\
& \frac{d V}{d t} \leq {\left[\left(S_{H}-S_{H}^{*}\right)+\left(E_{H}-E_{H}^{*}\right)+\left(I_{H}-I_{H}^{*}\right)+\left(R_{H}-R_{H}^{*}\right)+\right.} \\
&\left.\quad\left(S_{R}-S_{R}^{*}\right)+\left(I_{R}-I_{R}^{*}\right)\right] \cdot\left[-\mu\left(S_{H}-S_{H}^{*}\right)-\mu\left(E_{H}-E_{H}^{*}\right)\right. \\
&-\mu\left(I_{H}-I_{H}^{*}\right)-\mu\left(R_{H}-R_{H}^{*}\right)-\kappa\left(I_{H}-I_{H}^{*}\right)-(\delta+\phi) . \\
&\left.\left(S_{R}-S_{R} *\right)-(\delta+\phi)\left(I_{R}-I_{R}^{*}\right)\right] \\
& \leq-\mu\left(S_{H}-S_{H}^{*}\right)^{2}-\mu\left(S_{H}-S_{H}^{*}\right)\left[\left(E_{H}-E_{H}^{*}\right)+\left(I_{H}-I_{H}^{*}\right)\right. \\
&\left.+\left(R_{H}-R_{H}^{*}\right)\right]-\left(S_{H}-S_{H}^{*}\right) \cdot\left[\kappa\left(I_{H}-I_{H}^{*}\right)+(\phi+\delta) .\right. \\
&\left.\left(S_{R}-S_{R}^{*}+I_{R}-I_{R}^{*}\right)\right]-\mu\left(E_{H}-E_{H}^{*}\right)^{2}-\mu\left(E_{H}-E_{H}^{*}\right) \\
& {\left[\left(S_{H}-S_{H}^{*}\right)+\left(I_{H}-I_{H}^{*}\right)+\left(R_{H}-R_{H}^{*}\right)\right]-} \\
&\left(E_{H}-E_{H}^{*}\right)\left[\left(\kappa\left(I_{H}-I_{H}^{*}\right)+(\phi+\delta)\left(S_{R}-S_{R}^{*}+I_{R}-I_{R}^{*}\right)\right]\right. \\
&-\mu\left(R_{H}-R_{H}^{*}\right)^{2}-\mu\left(R_{H}-R_{H}^{*}\right) \cdot\left[\left(S_{H}-S_{H}^{*}\right)+\left(E_{H}-E_{H}^{*}\right)\right. \\
&\left.+\left(I_{H}-I_{H}^{*}\right)\right]-\left(R_{H}-R_{H}^{*}\right)\left[\left(\kappa\left(I_{H}-I_{H}^{*}\right)+(\phi+\delta)\right.\right. \\
&\left.\left(S_{R}-S_{R}^{*}+I_{R}-I_{R}^{*}\right)\right]-(\mu+\kappa)\left(I_{H}-I_{H}^{*}\right)^{2}-\mu\left(I_{H}-I_{H}^{*}\right) \\
& {\left[\left(S_{H}-S_{H}^{*}\right)+\left(E_{H}-E_{H}^{*}\right)+\left(R_{H}-R_{H}^{*}\right)\right]-\left(I_{H}-I_{H}^{*}\right) . } \\
& {\left[(\phi+\delta)\left(S_{R}-S_{R}^{*}+I_{R}-I_{R}^{*}\right)\right]-(\delta+\phi)\left(S_{R}-S_{R}^{*}\right)^{2} } \\
&-\mu\left(S_{R}-S_{R}^{*}\right) \cdot\left[\left(S_{H}-S_{H}^{*}\right)+\left(E_{H}-E_{H}^{*}\right)+\left(R_{H}-R_{H}^{*}\right)\right. \\
&\left.+\left(I_{H}-I_{H}^{*}\right)\right]-\left(S_{R}-S_{R}^{*}\right)\left[(\phi+\delta)\left(I_{R}-I_{R}^{*}\right)+\kappa\left(I_{H}-I_{H}^{*}\right)\right] \\
&-(\delta+\phi)\left(I_{R}-I_{R}^{*}\right)^{2}-\mu\left(I_{R}-I_{R}^{*}\right) \cdot\left[\left(S_{H}-S_{H}^{*}\right)+\right. \\
&\left.\left(E_{H}-E_{H}^{*}\right)+\left(R_{H}-R_{H}^{*}\right)+\left(I_{H}-I_{H}^{*}\right)\right]-\left(I_{R}-I_{R}^{*}\right) \\
& {\left[(\phi+\delta)\left(S_{R}-S_{R}^{*}\right)+\kappa\left(I_{H}-I_{H}^{*}\right)\right] }
\end{aligned}
$$


In view of the foregoing in Eq. (28), we have that $\frac{d V}{d t} \leq 0$, though $\frac{d V}{d t}=0$ only at $\mathcal{E}_{1}$ (i.e, if $S_{H}=S_{H}^{*}, E_{H}=$ $E_{H}^{*}, I_{H}=I_{H}^{*}, R_{H}=R_{H}^{*}, S_{R}=S_{R}^{*}$, and $\left.I_{R}=I_{R}^{*}\right)$. So, it follows from LaSalle's invariance principle [25] that all solution of system (1) approach $\mathcal{E}_{1}$ as $t \rightarrow \infty$ if $\mathcal{R}_{h}$ and $\mathcal{R}_{r}>1$. Therefore, the endemic equilibrium $\mathcal{E}_{1}$ is globally asymptotically stable in $\Omega$ whenever $\mathcal{R}_{h}$ and $\mathcal{R}_{r}>1$. This completes the proof $\square$.

\section{NumERICAL RESUlts AND DisCUSSION}

The sensitivity analysis of the basic reproduction number with respect to the model parameters is carried out and the numerical simulation of the model system (1) is done using Runge-Kutta fourth order scheme, using Matlab program to elucidate the qualitative analysis of the model system based on variables and parameter values presented in Table IV-A.

\section{A. Sensitivity Analysis}

Sensitivity analysis determined the behavior of the model in response to the changes in the variables and parameter values in the model structure. The contribution of parameters to the basic reproduction number reveals the effectiveness of each of the parameter value to persistence of the disease in the environment with respect to time. In the present case, the focus is given to determine how changes in the model parameters impact the effective reproduction number. However, this is done through the normalized forward-sensitive index.

The normalized forward-sensitive index of $\mathcal{R}_{h}$ and $\mathcal{R}_{r}$ to any parameter say (П) can be defined as

$$
\Gamma_{R_{h}}^{\aleph}=\frac{\partial R_{h}}{\partial \Pi} \times \frac{\Pi}{R_{h}}
$$

The sensitivity index of $\mathcal{R}_{h}$ with respect to parameters $\left(\beta_{2}, \gamma, \mu, \kappa\right.$, and $\left.\alpha\right)$ are as stated below:

For $\quad \beta_{2}, \quad \Gamma_{R_{h}}^{\beta_{2}}:=\frac{\partial R_{h}}{\partial \beta_{2}} \times \frac{\beta_{2}}{R_{h}}=1$

For $\quad \gamma, \quad \Gamma_{R_{h}}^{\gamma}:=\frac{\partial R_{h}}{\partial \gamma} \times \frac{\gamma}{R_{h}}=\frac{\mu}{\gamma+\mu}$

For $\quad \mu, \quad \Gamma_{R_{h}}^{\mu}:=\frac{\partial R_{h}}{\partial \mu} \times \frac{\mu}{R_{h}}=-\frac{\mu(\kappa+\alpha+2 \mu+\gamma)}{(\gamma+\mu)(\kappa+\alpha+\mu)}$

For $\quad \kappa, \quad \Gamma_{R_{h}}^{\kappa}:=\frac{\partial R_{h}}{\partial \kappa} \times \frac{\kappa}{R_{h}}=-\frac{\kappa}{\kappa+\alpha+\mu}$

For $\quad \alpha, \quad \Gamma_{R_{h}}^{\alpha}:=\frac{\partial R_{h}}{\partial \kappa} \times \frac{\kappa}{R_{h}}=-\frac{\alpha}{\kappa+\alpha+\mu}$

The sensitivity index of $\mathcal{R}_{r}$ with respect to parameters $(\tau, \delta \phi)$ are as stated below:

$$
\begin{array}{ll}
\text { For } \quad \tau, \quad \Gamma_{R_{r}}^{\tau}:=\frac{\partial R_{r}}{\partial \tau} \times \frac{\tau}{R_{r}}=1 \\
\text { For } \quad \delta, \quad \Gamma_{R_{r}}^{\delta}:=\frac{\partial R_{r}}{\partial \delta} \times \frac{\delta}{R_{r}}=-\frac{\delta}{\delta+\phi} \\
\text { For } \quad \phi, \quad \Gamma_{R_{r}}^{\phi}:=\frac{\partial R_{r}}{\partial \phi} \times \frac{\phi}{R_{r}}=-\frac{\phi}{\delta+\phi}
\end{array}
$$

The sensitivity indices of $\mathcal{R}_{h}$ and $\mathcal{R}_{r}$ with respect to each of the model parameters are presented in Tab. II based on parameter values from Tab. IV-A.
TABLE II

SENSITIVITy INDICES OF $\mathcal{R}_{h}$ AND $\mathcal{R}_{r}$ TO PARAMETERS

\begin{tabular}{c|l|l}
\hline Parameter & Description & Index \\
\hline \hline$\beta_{2}$ & Transmission rate & 1 \\
\hline$\gamma$ & Progression from $E_{H}$ to $I_{H}$ & 0.0001054 \\
\hline$\mu$ & Human natural death & -0.000175 \\
\hline$\kappa$ & Disease induced death rate & -0.0128 \\
\hline$\alpha$ & Progression from $I_{H}$ to $R_{H}$ & -0.9871 \\
\hline$\tau$ & Progression from $S_{R}$ to $I_{R}$ & 1 \\
\hline$\delta$ & Rodent natural death rate & -0.1177 \\
\hline$\phi$ & Rodent death due to predator & -0.8823 \\
\hline
\end{tabular}

In Tab. II, it is observed that the most sensitive parameters are $\left(\beta_{2}\right)$ and $(\tau)$ with both having sensitivity index of 1 . However,this implies that $\mathcal{R}_{h}$ or $\mathcal{R}_{r}$ approximately decreases by $1 \%$ when either $\beta_{2}$ or $\tau$ is decreased by 1 . This implies that any control measure that can reduced human transmission rate and progression of susceptible rodent to infected rodent will be a keynote in controlling the transmission dynamic of Lassa fever. Thus the parameter of model (1), their nominal value and references are presented in Tab. IV-A

TABLE III

Model Parameter VAlues

\begin{tabular}{l|l|l}
\hline Parameter & Nominal Value & Reference \\
\hline \hline$\beta_{2}$ & 0.05 & Assumed \\
\hline$\gamma$ & 0.52 & {$[5]$} \\
\hline$\Lambda_{H}$ & 0.215 & Assumed \\
\hline$\tau$ & 0.0003 & Assumed \\
\hline$\mu$ & 0.0000548 & {$[4]$} \\
\hline$\kappa$ & 0.01 & {$[4],[5],[13]$} \\
\hline$\alpha$ & 0.0005 & Assumed \\
\hline$\delta$ & 0.04 & {$[4],[5]$} \\
\hline$\phi$ & 0.01 & {$[5]$} \\
\hline$\Lambda_{R}$ & 0.00045 & Assumed \\
\hline$\beta_{1}$ & 0.00005 & {$[4]$} \\
\hline
\end{tabular}

The initial conditions $S_{H}(0)=111600000, E_{H}(0)=$ $63240000, I_{H}(0)=9300000, R_{H}(0)=186000, S_{R}(0)=$ 150000000 , and $I_{R}(0)=50000000$ where used for the simulation, noting the demographic population of Nigeria to be $186,000,000$ people. Using the above initial conditions and parameters' values in Tab. IV-A) to simulate model (1) to observe the behavior in each of the model compartments for scenarios with $\mathcal{R}_{0}>1$ and that with $\mathcal{R}_{0}<1$ as shown in (Fig. 2 - Fig. 7).

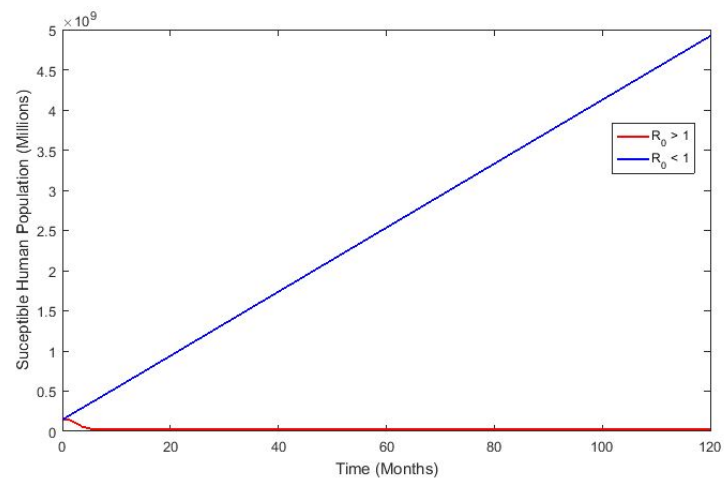

Fig. 2. Distribution of Susceptible Human Population 


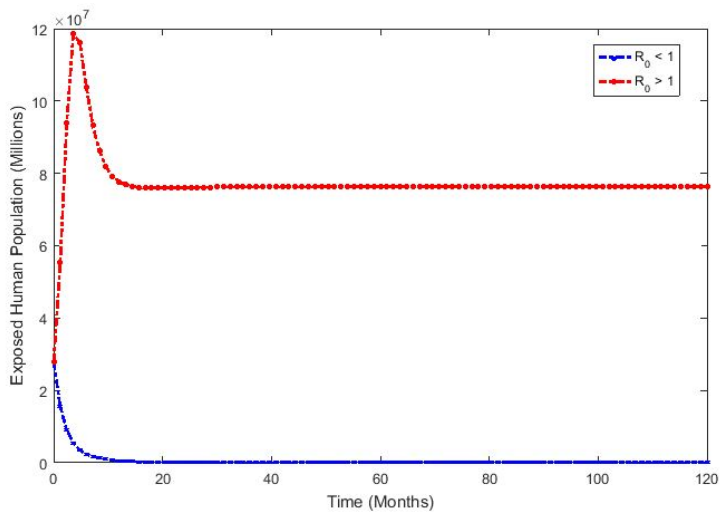

Fig. 3. Distribution of Exposed Human Population

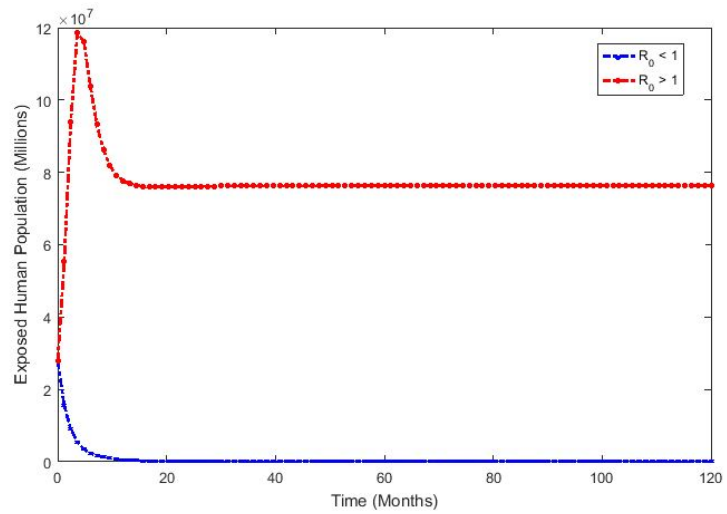

Fig. 4. Distribution of Infected Human Population

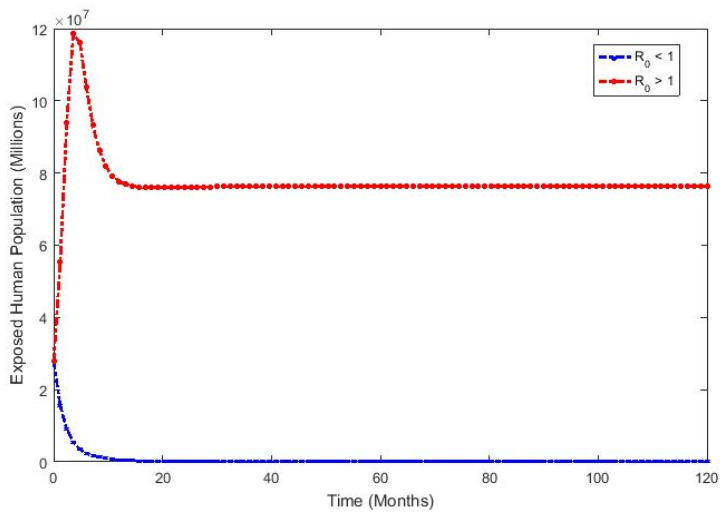

Fig. 5. Distribution of Recovered Human Population

Fig. 2-7 depict the behavior of model system (1) when the threshold parameter $\mathcal{R}_{0}=\max \left\{R_{h}=0.1653, R_{r}=\right.$ $0.1400\}<1$, and $\mathcal{R}_{0}=\max \left\{R_{h}=1.1568, R_{r}=\right.$ $12.3733\}>1$, using parameters in table (IV-A)

Fig. 2 depicts the evolution of susceptible humans which illustrate increase in human population when $\mathcal{R}_{0}<1$ and steady decline when $\mathcal{R}_{0}>1$. Fig. 3 describe the evolution of exposed humans to Lassa disease when $\mathcal{R}_{0}<1$ and $\mathcal{R}_{0}>1$. Fig. 4 describe the evolution of infected humans. This shows that when $\mathcal{R}_{0}>1$, there is a steady increase in the infected population over time until it reaches its peak, after which slowly decline to a stable population level. On the contrary, When $\mathcal{R}_{0}<1$, there is a very slight increase is number of infected individuals which is immediately followed by a steady and rapid drop in the population as it eventually approach the zero population level. Also, Fig. 5 depicts the profile of the recovered human population when $\mathcal{R}_{0}<1$ and when $\mathcal{R}_{0}>1$.

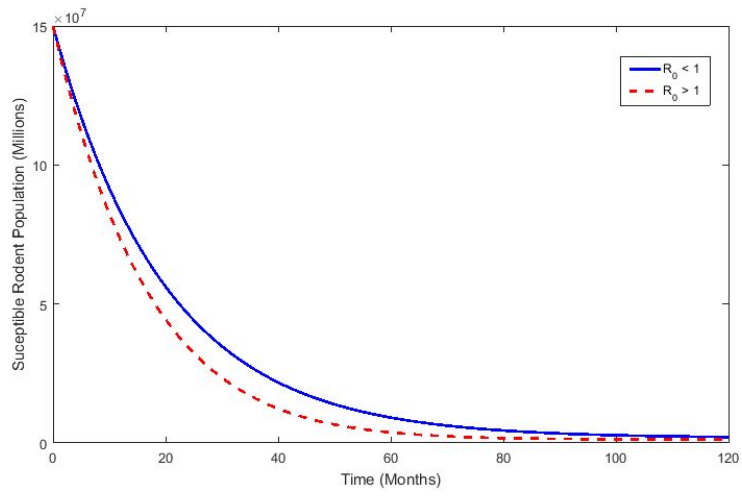

Fig. 6. Distribution of Susceptible Rodent Population

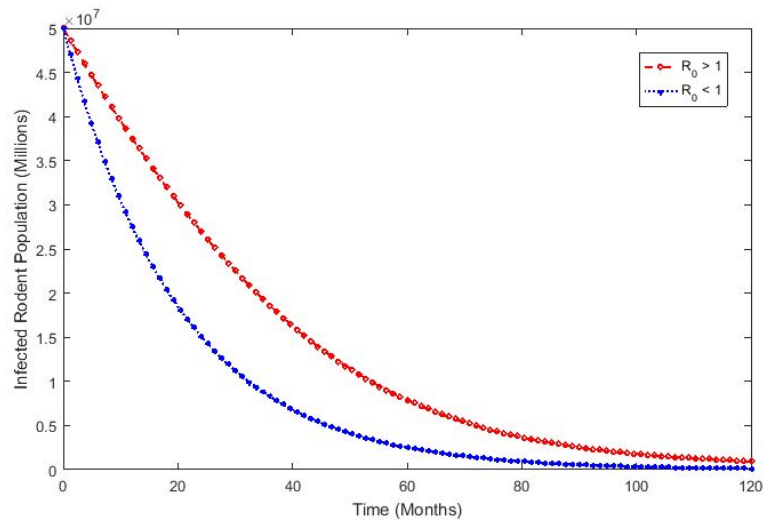

Fig. 7. Distribution of infected Rodent Population

However, Fig. 6 describes the evolution of susceptible rodents' population and this shows that when $\mathcal{R}_{0}>1$ there was a drastic decline in susceptible rodents population compared with when $\mathcal{R}_{0}<1$, while Fig. 7 describes the evolution of infected rodents' population when $\mathcal{R}_{0}<1$, and this reveals a sharp decline in infected rodents population compared with when $\mathcal{R}_{0}>1$,Thus, the basic reproduction number $\mathcal{R}_{0}$, is shown to be a distinguishing threshold parameter of the extinction or persistence of Lassa fever disease.

In order to investigate further into the disease transmission dynamics, we perform plots for new cases of Lassa disease infection as depicted in 8 and 9 .

Fig. 8 was simulated using parameter value in table (IV-A) with $\beta_{2}$ taken as $(0.5,0.51 \ldots . .0 .6,1,1.1, \ldots, 1.5)$ which reveals the effects of varying the human to human contact rate on model system (1). This depicts that increase in the value of $\left(\beta_{2}\right)$ yields increase in the number of new infected humans. 


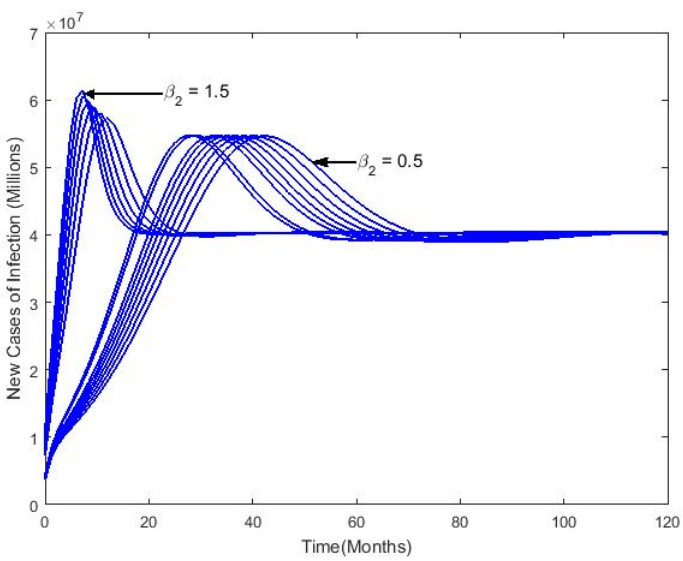

Fig. 8. Simulation of New-Cases of Lassa Fever infection

However from our simulation, increasing human contact rate say [ $\beta_{2}=1.5$ ] produces a sharp increase in the population of infected individuals having high saturation within a very short time, after which it slowly declines to its equilibrium. Thus, decreasing the value of $\beta_{2}$ say [ $\left.\beta_{2}=0.5\right]$ elapse the time for new cases of infection to reach its peak (Prolong rapid spread of the disease) and also increases time to attain stability.

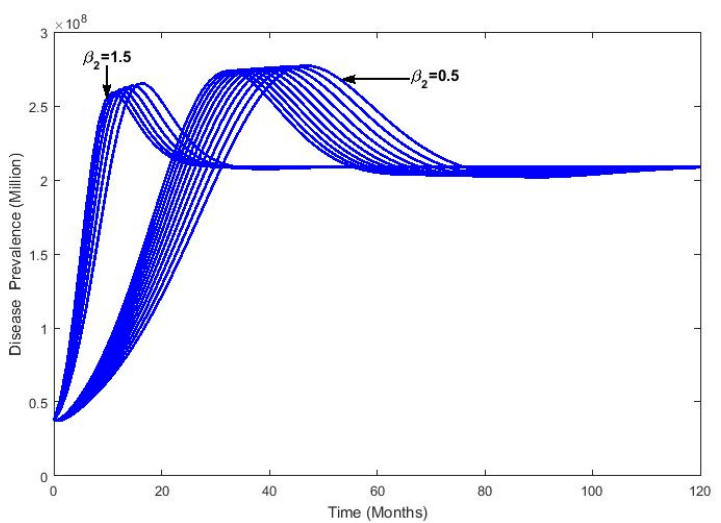

Fig. 9. Simulation of Lassa Fever Disease Prevalence

Fig. 9 depicts the prevalence of Lassa fever in model (1), which reveals that increase in the force of human to human contact [say $\beta_{2}=1.5$ ] reduces the time prevalence of Lassa Fever reaches saturation (Peak) infected individuals. However, by decreasing the value of $\beta_{2}$ say $\left[\beta_{2}=0.5\right]$, Lassa virus disease infects more individuals within long duration of time compared to when there is an increase in the number of human to human contact. By implication, if a control measure is instilled on mitigating increase in force of infection $\left(\beta_{2}\right)$, this will allow time for policy maker and health sector to provide a lasting solution (cure or Vaccine) to the spread and demise of Lassa Fever.

To further understand the transmission dynamic of Lassa fever disease, three different cases were consider by varying the effective human to human contact rate $\left(\beta_{2}\right)$ and rodent to rodent contact rate $(\tau)$ with parameter values in table (IV-A) for simulation and to determine the eventual behavior of the infected compartment over time as depicted in Figure (10-12). Moreover, this analysis illustrate a different approached as to work done in [4]-[6], [23].

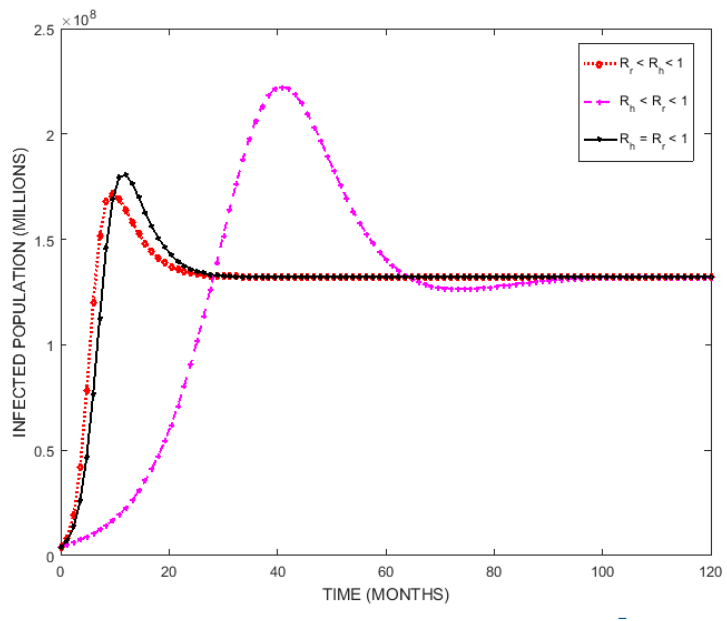

Fig. 10. Case 1

The scenario where both human and rodent reproduction number are less than unity is depicted in Figure (10) with $\beta_{2}=3,0.6,2.13$ and $\tau=0.003,0.0027,0.002$ (so that $\mathcal{R}_{r}=0.340<\mathcal{R}_{h}=0.9016<1, \mathcal{R}_{h}=0.198<\mathcal{R}_{r}=$ $0.940<1$, and $\mathcal{R}_{r}=0.7040=\mathcal{R}_{h}=0.7040<1$ ) other parameter value used are in Table (IV-A). The impact on infected population when $\left(\mathcal{R}_{r}<\mathcal{R}_{h}\right)$ is more pronounced as shown in figure (10), the infected population reaches its climax of infection faster before decreasing gradually until it reaches stability compared with when $\left(\mathcal{R}_{h}<\mathcal{R}_{r}\right)$ and $\left(\mathcal{R}_{h}=\mathcal{R}_{r}\right)$. Conversely, when $\left(\mathcal{R}_{h}<\mathcal{R}_{r}\right)$ there was a steady growth in the infected population until it reaches its peak before declining to attained stability. Case 2: represented

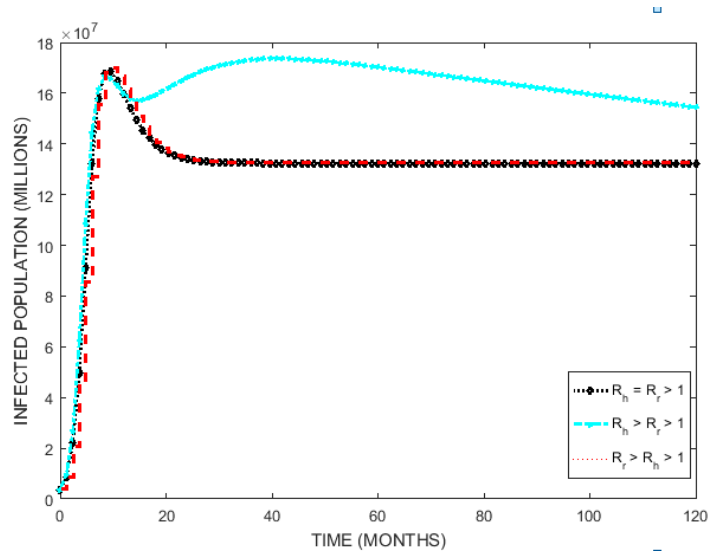

Fig. 11. Case 2

in Figure (11) depicts the scenario where both human and rodent reproduction number are greater than unity, by varying parameter: $\beta_{2}=3.2,4,3.38$ and $\tau=0.5,0.003,0.00323$ with parameter value used in Table (IV-A), so that $\mathcal{R}_{r}=166.707>$ $\mathcal{R}_{h}=1.058>1, \mathcal{R}_{h}=1.190>\mathcal{R}_{r}=1.0400>1$, and $\mathcal{R}_{r}=1.117=\mathcal{R}_{h}=1.117>1$. Since Lassa fever is zoonotic, there is a high prevalence when $\mathcal{R}_{h}>\mathcal{R}_{r}>1$ 
as against when $\mathcal{R}_{h}=\mathcal{R}_{r}>1$. This is a pointer that can be useful to proposed optimal intervention strategies in mitigating the spread of Lassa disease.

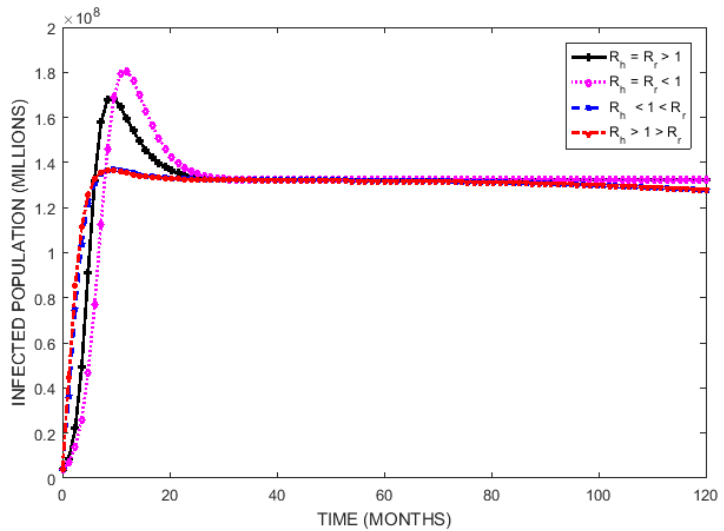

Fig. 12. Case 3

Figure 12 represent a complete scenario using different values for $\beta_{2}=4,3,2.13,3.38$ and $\tau=0.0006,0.5,0.00323,0.002$, with parameter values in Table (IV-A) so that $\left(\mathcal{R}_{r}=19.831>\right.$ $\left.1 \& \mathcal{R}_{r}=0.640<1\right),\left(\mathcal{R}_{h}=0.9016<1 \& \mathcal{R}_{r}=50.040>1\right)$, $\left(\mathcal{R}_{h}=0.640 \& \mathcal{R}_{r}=0.640<1\right)$ and $\left(\mathcal{R}_{h}=10.047 \& \mathcal{R}_{r}=\right.$ $10.047>1$. By investigating the impart of reproduction number on the population, it could be established that when $\max \left\{\mathcal{R}_{0}\right\}>1$, Lassa fever is endemic and reaches stability faster, this implies that when human transmission rate is high the spread of Lassa disease will be pandemic and this can be controlled through awareness and government intervention schemes as noted by [26].

\section{CONCLUSION}

An improved deterministic model for the transmission dynamics of Lassa fever is developed and rigorously analyzed. The analysis and simulations of the model reveal the followings: $\mathrm{i}$

1) The Model (1) has a locally and globally asymptotically stable disease-free equilibrium whenever the associated reproduction number $\left(\mathcal{R}_{0}\right)$ is less than unity and unstable when the associated basic reproduction number $\left(\mathcal{R}_{0}\right)$ is greater than unity;

2) Moreover, in addition to the disease-free equilibrium, the model has another equilibrium solution called the endemic equilibrium which is unique whenever $\mathcal{R}_{0}>1$. Thus, control measures must be implemented to consistently keep $\mathcal{R}_{0}$ below unity so that the disease spread can be under check;

3) The sensitivity analysis of the model parameters reveals that the most sensitive parameter are that of the human transmission rate and progression rates of rodents to the infected compartment. Thus, any control measure that can significantly reduce these parameter and also increase rodents' death by predator will be effective in eradicating the disease from the population;

4) The numerical solution of the model reveals that for Lassa fever not to be endemic in the population, the effective contact rate of human to human should be minimize and this can be achieved through engaging communities to promote desired personal hygiene cum safe health practices among populace, environmental hygiene, and providing accurate and timely health advice and information on the disease.

\section{REFERENCES}

[1] F. Brauer, C. Castillo-Chavez, A. Mubayi, S. Towers, "Some models for epidemics of vector-transmitted diseases", Infectious Disease Modelling, KeAi Advancing Research Evolving Science, vol. 1(2016), pp.79-87, 2016. http://dx.doi.org/10.1016/j.idm.2016.08.001

[2] T. S. Faniran, "A Mathematical Modelling of Lassa Fever Dynamics with Non-drug Compliance Rate", International Journal of Mathematics Trends and Technology, vol. 47(5), pp. 305-318, 2017.

[3] I. S. Lukashevich, "The Search for Animal Models for Lassa Vaccine Development", National Institutes of Health, vol. 12(1), pp.71-86, 2013.

[4] D. Omale and T. E. Edibo, "Mathematical Models for Lassa Fever Transmission with Control Strategies", Computing, Information System, Development Informatics \& Allied Research Journal, vol.6(4), pp. 2532, 2015.

[5] S. Usman. and I. Isa Adamu, "Modelling the Transmission Dynamics of the Lassa Fever Infection",IISTE, vol.8(5), pp.42-63, 2018.

[6] J. O. Akanni and A. D. Adedipo, "Sensitive Analysis of the Dynamical Transmission and Control of Lassa Fever Virus", Asian Research Journal of Mathematics, vol. 9(3), pp. 1-11, 2018.

[7] M. M. Ojo, and F. O. Akinpelu, "Lyapunov Functions and Global Properties of SEIR Epidemic Model", International Journal of Chemistry, Mathematics and Physics, vol.1(1), pp.11-16, 2017.

[8] B. I. Eraikhuemen and O. Eguasa,'Lassa fever and its Control Measures", Journal of Natural Sciences Research,vol. 7(12), pp. 75-79, 2017.

[9] Lassa Fever Outbreak Situation Report, "Nigeria Center For Disease Control", [Cited 2019 Mar 11]. http://www.ncdc.gov.ng serial number:04.

[10] L. Cai and X. Li, "Analysis of a Simple Vector-Host Epidemic Model with Direct Transmission", Discrete Dynamics in Nature and Society, vol. 2010, Article ID 679613, 12 pages, 2010. https://doi.org/10.1155/2010/679613

[11] W. O. Kermack, and A. G. McKendrick, "A contribution to the mathematical theory of epidemics",Proc. Roy. Soc. London, vol. 115, pp. 700-721, 1927.

[12] J B McCormick, I J King, P A Webb, C L Scribner, R B Craven, K M Johnson, L H Elliott, and R Belmont-Williams, "Lassa fever. Effective therapy with ribavirin", N Engl J Med. 1986 vol. 314(1), pp.20-6, 1986. doi:10.1056/NEJM198601023140104, PMID: 3940312.

[13] Viral Hemorrhagic Fever (VHFs), "Centers for Disease Control and Prevention", [visited march 2019], https://www.cdc.gov/vhf/virusfamilies/arenaviridae.html

[14] E. Fichet-Calvet, E. Lecompte, L. Koivogui, B. Soropogui, A. Doré, F. Kourouma, O. Sylla, S. Daffis, K. Koulémou, and J. Ter Meulen, "Fluctuation of abundance and Lassa virus prevalence in Mastomys natalensis in Guinea, West Africa", Vector Borne Zoonotic Dis., vol. 7(2), pp. 119-28, 2007. doi:10.1089/vbz.2006.0520, PMID: 17627428.

[15] World Health Organization, "Lassa Fever-fact sheet, reviewed July 2017”, [visited march 2019] http://www.who.int/mediacentre/factsheets/fs179/en/

[16] R. M. Anderson and R. M. May, "Infectious Disease of Humans", Oxford University Press, Oxford, 1991.

[17] L. Esteva and C. Vargas, "Analysis of a dengue disease transmission model", Mathematical biosciences, vol. 150(2), pp.131-151, 1998.

[18] G. Cruz-Pacheco, L. Esteva, J. A. Montaño-Hirose, C. Vargas, "Modelling the dynamics of West Nile Virus", Bulletin of mathematical biology, vol. 67(6), pp. 1157-1172, 2005. https://doi.org/10.1016/j.bulm.2004.11.008

[19] B. Traoré, B. Sangaré, S. Traoré, "A Mathematical Model of Malaria Transmission with Structured Vector Population and Seasonality", Journal of Applied Mathematics, vol. 2017, Article ID 6754097, 15 pages, 2017. https://doi.org/10.1155/2017/6754097

[20] Lassa Fever, "Emergencies preparedness, response", 2020 https://www.who.int/csr/don/20-february-2020-lassa-fever-nigeria/en/

[21] P. Van den Driessche, and J. Watmough, "Reproduction numbers and sub-threshold endemic equilibria for compartmental models of diseases transmission", Mathematical Biosciences, vol. 180, pp. 29-48, 2002. 
[22] J. Lassale, and S. Lefschetz, "The stability of dynamical system", SIAM Philadelphia, 2010.

[23] O. S. Obabiyi, and A. O. Akindele, "Mathematical model for lassa fever transmission with variable human and reservoir Population", International Journal of Differential Equation and Applications, vol. 16(1), pp. 67-91, 2017.

[24] S. O. Adewale, I. A. Olapade, S. O. Ajao, G. A. Adeniran, and O. T. Oyademi, "Mathematical Analysis of Lassa Fever Model with Isolation. Asian Journal of Natural \& Applied Sciences", vol. 5(3), pp. 47-57, 2016.

[25] J. Lasalle. "The stability of dynamical systems" Philadelphia: SIAM, 1976.

[26] E. A. Bakare, E. B. Are, O. E. Abolarin, S. A. Osanyinlusi, Benitho Ngwu, Obiaderi N. Ubaka, "Mathematical Modelling and Analysis of Transmission Dynamics of Lassa Fever", Journal of Applied Mathematics, vol. 2020, Article ID 6131708, 18 pages, 2020 https://doi.org/10.1155/2020/6131708 